\title{
Brief pain re-assessment provided more accurate prognosis than baseline information for low-back or shoulder pain
}

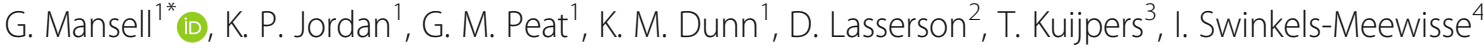
and D. A. W. M. van der Windt ${ }^{1}$

\begin{abstract}
Background: Research investigating prognosis in musculoskeletal pain conditions has only been moderately successful in predicting which patients are unlikely to recover. Clinical decision making could potentially be improved by combining information taken at baseline and re-consultation.

Methods: Data from four prospective clinical cohorts of adults presenting to UK and Dutch primary care with low-back or shoulder pain was analysed, assessing long-term disability at 6 or 12 months and including baseline and 4-6 week assessments of pain. Baseline versus short-term assessments of pain, and previously validated multivariable prediction models versus repeat assessment, were compared to assess predictive performance of long-term disability outcome. A hypothetical clinical scenario was explored which made efficient use of both baseline and repeated assessment to identify patients likely to have a poor prognosis and decide on further treatment.

Results: Short-term repeat assessment of pain was better than short-term change or baseline score at predicting long-term disability improvement across all cohorts. Short-term repeat assessment of pain was only slightly more predictive of long-term recovery (c-statistics $0.78,95 \% \mathrm{Cl} 0.74$ to 0.83 and $0.75,95 \% \mathrm{Cl} 0.69$ to 0.82 ) than a multivariable baseline prognostic model in the two cohorts presenting such a model (c-statistics $0.71,95 \% \mathrm{Cl} 0.67$ to 0.76 and 0.72 , $95 \% \mathrm{Cl} 0.66$ to 0.78 ). Combining optimal prediction at baseline using a multivariable prognostic model with short-term repeat assessment of pain in those with uncertain prognosis in a hypothetical clinical scenario resulted in reduction in the number of patients with an uncertain probability of recovery, thereby reducing the instances where patients may be inappropriately referred or reassured.
\end{abstract}

Conclusions: Incorporating short-term repeat assessment of pain into prognostic models could potentially optimise the clinical usefulness of prognostic information.

Keywords: Prognosis, Primary care, Consultation, Musculoskeletal conditions, Low back pain, Shoulder pain

\section{Background}

Musculoskeletal conditions account for $14 \%$ of all general practice (GP) consultations, with a quarter of the registered population consulting each year with a musculoskeletal problem [1]. Regional, non-inflammatory disorders - back, knee, neck and shoulder pain - are among the most common problems, together representing more than $40 \%$ of all consultations for musculoskeletal

\footnotetext{
* Correspondence: g.mansell@keele.ac.uk

${ }^{1}$ Arthritis Research UK Primary Care Centre, Research Institute for Primary Care and Health Sciences, Keele University, Keele, Staffordshire ST5 5BG, UK Full list of author information is available at the end of the article
}

conditions [1]. Outcome varies considerably between individual patients [2-5] and remains difficult to predict accurately despite a considerable number of published studies deriving prognostic models [6]. This has led to uncertainty around how to manage patients who present to GPs with musculoskeletal pain. Such uncertainty may result in patients being referred unnecessarily, or being incorrectly reassured that their pain will subside when they might in fact benefit from early treatment.

Although the methods for prognostic model research have advanced over the past 10 years, little research has addressed issues related to the clinical usefulness and 
impact of prognostic information. A feature of most published studies in this field has been the reliance on information on prognostic factors assessed at one point in time (baseline), with prediction of future outcome based on this one assessment only. However, prognostic information will often include time-varying factors, and using information from a single assessment only is likely to result in suboptimal prognostic performance as patients tend to consult when their symptoms are at their worst, and short-term improvements following the consultation are likely [7]. These short-term improvements in pain episodes have been described not only in acute low back pain (e.g. [8]) but also in longerterm pain problems $[9,10]$. A single assessment also seems out of touch with clinical practice where GPs incorporate information from previous visits to estimate prognosis and decide on treatment and referral. When a patient first consults with musculoskeletal pain it is not always certain whether they require immediate referral, can be reassured that their pain will subside, or require monitoring over time to see how the problem develops; further information gained from repeat consultations may help with such decisions. This implies that information collected at the time of the first consultation may not provide optimal prognostic information. Indeed, it has been previously shown that combining information on prognostic factors taken at baseline and short-term repeated assessment [11] provided better prediction of long-term outcome in low back pain patients than a baseline score. However, this does not address the issue of how to manage patients who re-attend primary care for musculoskeletal pain, or whether brief repeated assessments of symptoms provide more information about likely future outcomes compared to a more comprehensive baseline assessment. Research to address these questions might aid clinical decision making regarding the need for early referral and treatment in patients with a low probability of recovery, whether monitoring of symptoms might be helpful, or whether patients can be reassured regarding their prognosis. The aim of this study was therefore to explore whether the efficiency of clinical decision making can potentially be improved by combining information taken at baseline and re-consultation, as identifying optimal approaches to collecting prognostic data could lead to improved estimation of long-term outcomes, providing better information to support treatment decisions.

We hypothesised that incorporating information from repeated assessments of pain in prognostic models will significantly improve prognostic performance and reduce uncertainty in clinical decision making when estimating the likelihood of long-term outcomes in patients consulting with musculoskeletal pain.

\section{Specific objectives}

1. To explore the prognostic performance of repeated (4-6 week) assessment or change in pain compared with baseline assessment only in the prediction of longer-term (6-12 month) outcome in painful musculoskeletal conditions, and evaluate the consistency of the findings across different samples;

2. To assess the prognostic performance of the incorporation of short-term repeated assessment of pain with data collected on multiple prognostic factors at baseline, and assess the unique contribution of short-term repeat assessment of pain, in the prediction of long-term outcome;

3. To test a strategy for accurately and efficiently classifying patients as being at low or high probability of recovery, based on prognostic information measured at a single point in time in combination with a short-term repeated assessment in those at intermediate risk of a poor prognosis at baseline.

\section{Methods}

Potentially eligible studies were identified from two bibliographic databases held at Keele University that include the results of sensitive searches for prognosis studies in primary care. A systematic search of eligible studies was not conducted; the aim was instead to identify studies of different musculoskeletal conditions that met specific criteria, allowing us to test the study hypothesis. First authors or principal investigators of eligible studies were contacted to ask for their collaboration and request necessary data.

\section{Inclusion criteria}

- Design: prospective cohort study;

- Population: patients consulting with spinal pain (back or neck), shoulder pain, or knee pain in primary care;

- Outcome measures: pain-specific disability (e.g. Roland-Morris Disability Questionnaire (RMDQ) [12] or Shoulder Disability Questionnaire (SDQ) [13]), patient-perceived recovery;

- Prognostic factor measurement: at least two assessments of pain: at baseline and short-term (within 4-6 weeks of baseline assessment);

- Outcome ascertainment: Longer-term follow-up (at least 6 months after baseline assessment).

\section{Statistical Analysis}

Descriptive statistics were used to characterise each of the study samples, present long-term outcome scores for disability, and present short-term changes and repeat 
assessment scores in pain. Baseline and short-term pain scores were analysed on their original scale (e.g. visual analogue scale or $0-10$ numeric rating scale [14]).

\section{Objective 1: Comparison of repeat assessment with baseline value}

The long-term recovery outcome for objective 1 was defined based on a validated cut-off point for minimal important change in disability for the RMDQ and SDQ (at least $30 \%$ improvement $[15,16]$ ) allowing classification of participants into those "Improved" or "Not improved".

For each study, pain measured at baseline was entered into separate logistic regression models with the dichotomised variable for disability improvement as the outcome measure, to investigate whether this baseline variable predicted improvement in disability at longterm follow-up. The same analysis was then repeated using short-term change (difference between baseline and shortest follow-up point from each study) and absolute short-term follow-up score (repeat assessment) of pain as predictors of disability improvement at longterm follow-up, in separate analyses. The strength of association (odds ratios (ORs) with 95\% CI), model goodness-of-fit (Hosmer \& Lemeshow and Nagelkerke pseudo r-square), and prognostic performance (AUC (cstatistic)) were compared for each model [17]. A cstatistic of 0.75 or above was considered to indicate good discrimination (i.e. a high level of sensitivity and specificity), and a value of 0.50 indicates a level of association no better than chance [17]. Differences in c-statistics were compared using the STATA roccomp command
[18]. A $p$ value of $<0.05$ indicates a statistically significant difference in the c-statistics.

\section{Objective 2: Comparison of repeat assessment with multivariable baseline prognostic model}

Only datasets that had already obtained a prediction model (van der Windt [19] and Kuijpers [20] - both shoulder pain populations) were used in this analysis. The outcome measure used was patient perceived recovery from shoulder symptoms (dichotomised: $1=$ recovery) as used in the original prognostic model, rather than the disability improvement scores used in objective 1. Each of the prognostic models were rerun to check whether they produced the same results as the original study. Prognostic performance was then described and compared to that of incorporating a brief repeat assessment of pain in the same way as in Objective 1 (Hosmer \& Lemeshow test, Nagelkerke pseudo R-square, ORs and AUC), using the STATA roccomp command to test for statistically significant differences in c-statistics between the models. We also examined the contribution of repeat assessment of pain when adding this to the original prognostic model using an omnibus test [21] to examine whether adding such short-term information significantly improved fit of the prediction model.

\section{Objective 3: Scenario combining optimal baseline prediction with brief repeated assessment}

The purpose of this final objective was to test a more efficient hypothetical clinical scenario (Fig. 1) using the

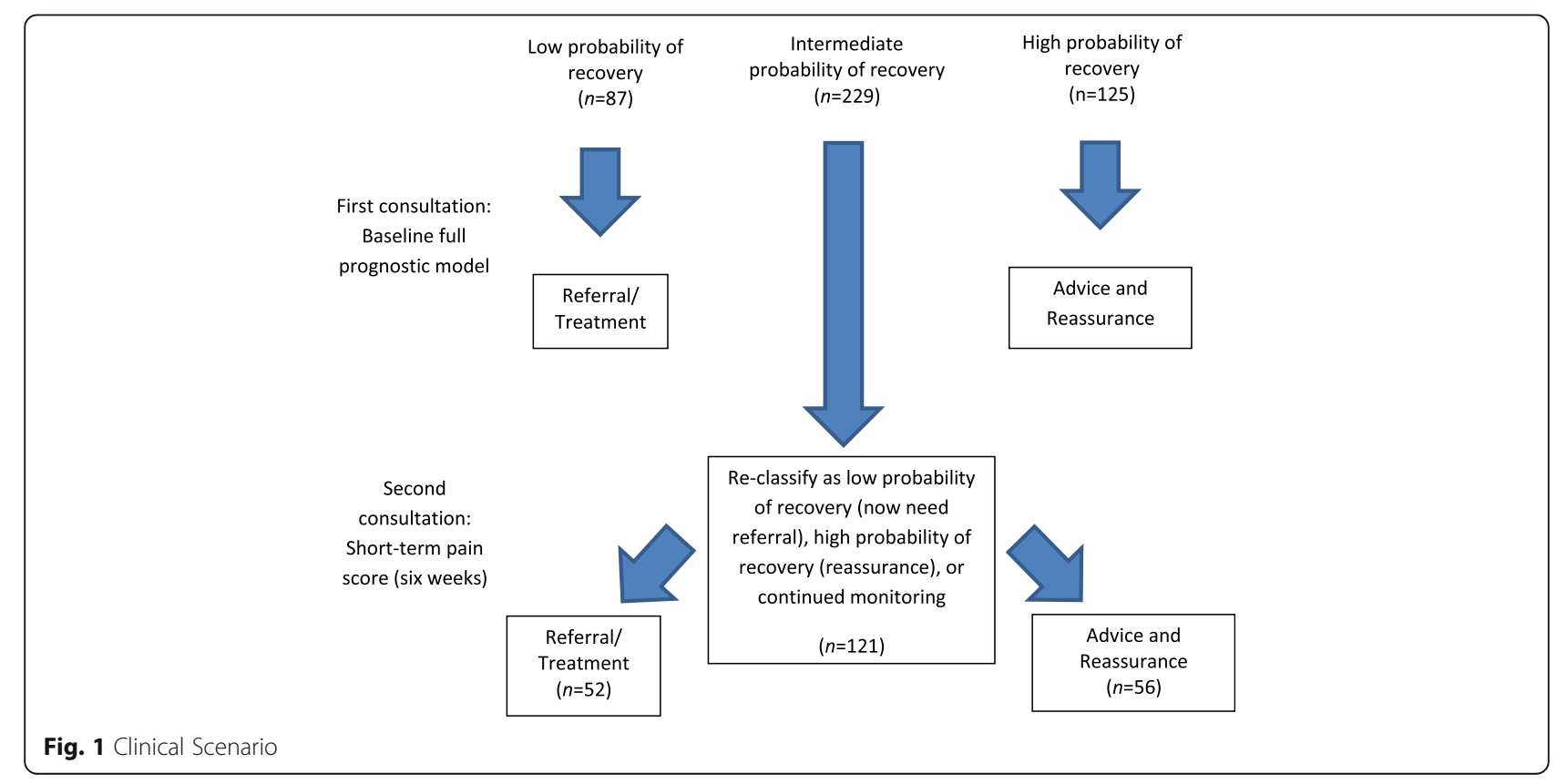


Kuijpers [20] dataset, that does not require all patients to receive a repeat assessment. The scenario aims to discriminate between those with a high probability of recovery (i.e. likely to need advice and pain relief but no further monitoring required) and those with a low probability of recovery at six months (i.e. likely to require treatment or referral). Prognostic information collected at baseline in all patients was combined with a short-term repeat assessment of pain in those with an intermediate probability of recovery (uncertain prognosis). This scenario would result in only those who GPs were most uncertain about being asked to return for a repeat consultation, who would then be re-assessed and a better informed decision made based on the patient's reporting of pain at that second consultation.

Patient perceived recovery at six months was used as the outcome measure (as in objective 2). Two stages of analyses were performed to assess prognostic performance of the combined strategy:

1. Discrimination at baseline between those at low, intermediate, and high probability of long-term recovery using cut-points based on predicted probabilities of recovery (scores of $\leq 0.33,0.34$ to $0.66, \geq 0.67)$ from the optimal baseline prognostic model. Cut-offs for what constituted low, intermediate and high probability of recovery were chosen based on consensus among the clinicians and methodologists within the study team, given the absence of empirically derived cut-offs in this area.

2. Discrimination at short-term follow-up in those with intermediate probability of long-term recovery ( $n=229)$ by reclassification as low, intermediate, and high probability of recovery at six months based on the results of the full baseline model plus a repeat assessment of pain six weeks after their baseline assessment. Cut-off points for predicted probabilities were the same as those used in step 1 .

The results of these two stages were then compared to assess how many patients originally allocated to the intermediate probability (uncertain) group (stage 1) had moved to either the high or low probability group by short-term follow-up (stage 2), and how many of these were correctly or incorrectly classified in terms of their long-term outcomes.

\section{Results}

\section{Descriptive Results}

Four datasets were identified, requested and obtained $[19,20,22,23]$. The design and results of these studies are summarised in Table 1 . Two included patients with low back pain and two included patients with shoulder pain. No studies that included patients with knee pain were considered eligible for this study. The disability outcome measures used for these populations were the Roland-Morris Disability Questionnaire (RMDQ) [12] and Shoulder Disability Questionnaire (SDQ) [13] respectively. All studies recruited patients from primary care (general practice) clinics. One study [23] only included patients who had experienced pain for a month or less and two papers included patients with new pain episodes [19, 20]. The Dunn study [22] included a mix of patients with pain of short- and longer-term duration. All studies showed mean improvement in the outcome of disability, and $24 \%$ to $64 \%$ achieved $30 \%$ improvement in disability at the long-term follow-up point. The studies measured a number of different prognostic factors, including pain duration, fear-avoidance beliefs, [20, 22, 23] pain catastrophising [22] and pain coping [22]. Two studies $[19,20]$ had previously tested prognostic models within these datasets. Full details about the measures used in each study can be found in Additional file 1 .

\section{Objective 1}

This objective compared the performance of the different models in predicting long-term disability outcome: (1) baseline pain scores only, (2) short-term pain score (repeat assessment at short-term follow-up), and (3) short-term change in pain (change since baseline). The Hosmer \& Lemeshow tests indicated good fit for nearly all analyses (Table 2). The Nagelkerke $R^{2}$ values were higher for the repeat assessment of pain models. The cstatistics (AUC) also suggest that a repeat assessment of pain at short-term follow-up best predicts the outcome of disability improvement, followed by the short-term change models. For three of the datasets (Dunn, [22] Kuijpers, [20] and Swinkels-Meewisse [23]), statistically significant differences $(p<0.05)$ were found between the $c$-statistics for the models using baseline pain and repeat assessment score. A statistically significant difference in c-statistics was found between repeat assessment score and change in pain in two of the datasets (SwinkelsMeewisse [23] and Van der Windt [19]). Overall, repeat assessment of pain appeared to best predict disability outcome.

\section{Objective 2}

In the two datasets used for objective 2 (Van der Windt [19] and Kuijpers [20]), the number of people reporting recovery was 53\% $(n=232)$ in the Kuijpers [20] dataset and $66 \%(n=218)$ in the Van der Windt [19] dataset. When the models (full prediction model at baseline, and full prediction model incorporating repeat assessment of pain) for each dataset were compared, the c-statistics indicated that the addition of repeat assessment of pain was slightly more predictive compared to the full baseline models only, with the difference in predictive 


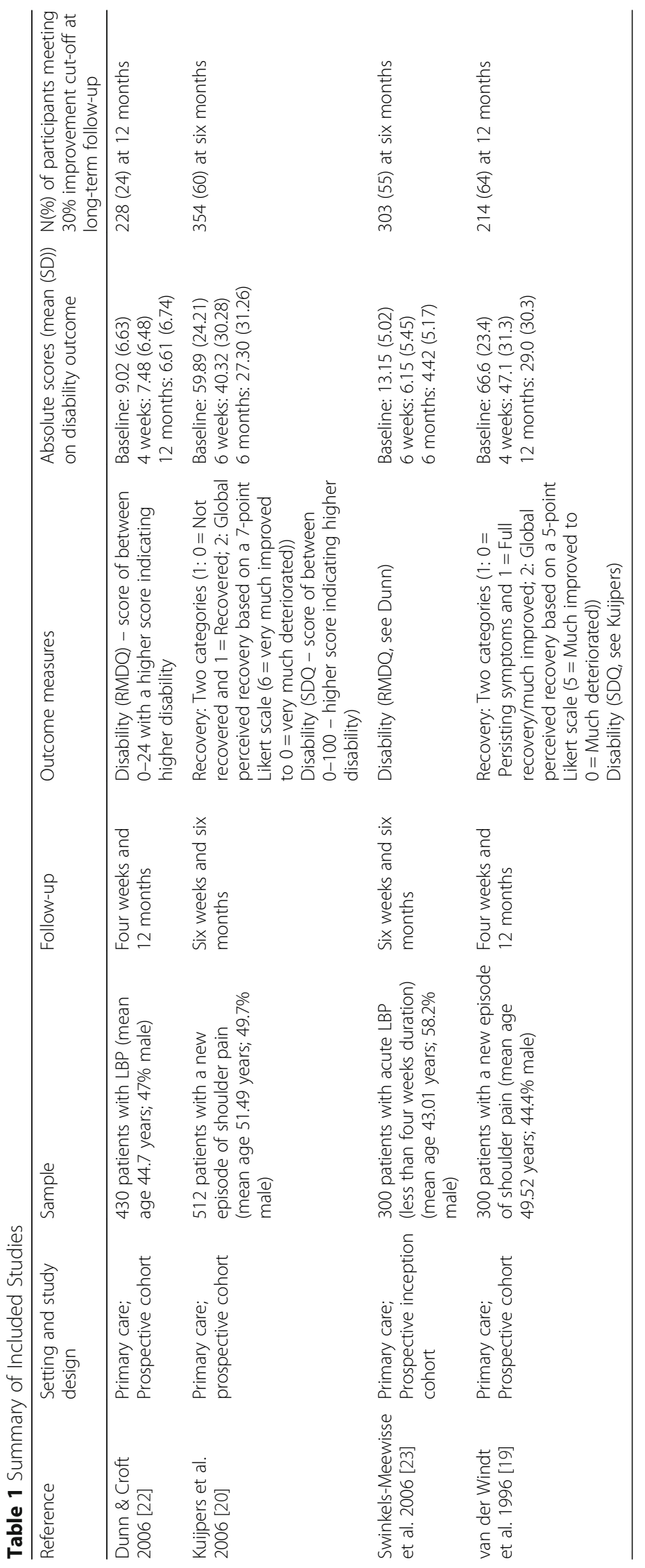




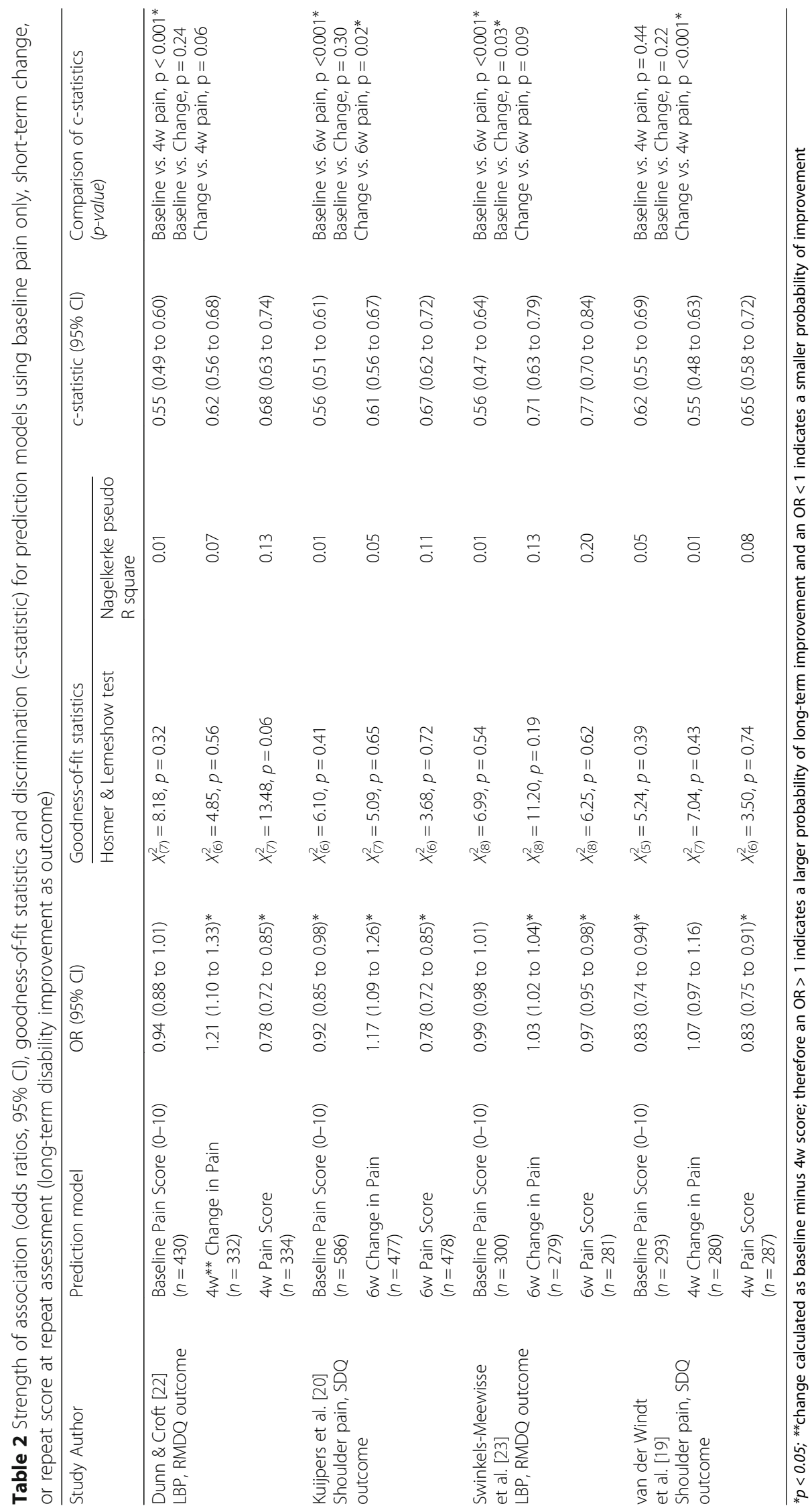


performance statistically significant $(p<0.05)$ for the Kuijpers dataset (c-statistic $0.78,95 \%$ CI 0.74 to 0.83 versus $0.71,95 \%$ CI 0.67 to 0.76 ), but not for the Van der Windt dataset (c-statistic $0.75,95 \%$ CI 0.69 to 0.82 versus $0.72,95 \%$ CI 0.66 to 0.78 , see Table 3 ). The omnibus tests for both datasets indicate that the addition of repeat assessment of pain (Kuijpers $X_{(6)}^{2}=109.23, p<0.05$; van der Windt $\left.X_{(4)}^{2}=35.00, p<0.05\right)$ to the full baseline prognostic model significantly improve the fit of the model.

\section{Objective 3}

The purpose of this final objective was to explore the hypothetical clinical scenario, aiming to combine optimal baseline data with follow-up assessment data in a subset of participants with uncertain prognosis, which would make efficient use of the repeated assessment. The full prediction model was found to provide good prognostic performance at baseline in the Kuijpers [20] dataset (Table 3). Table 4 shows the estimated frequencies of the low, intermediate and high probability of recovery groups in the two stages, and their observed outcome frequencies. Figure 1 presents the numbers in the context of the scenario for clarity.

In stage 1 , the full model at baseline presentation leaves 229 (52\%) participants in the uncertain (intermediate) category. Repeat assessment of pain at six weeks in this intermediate group reduces this number to $121(27.4 \%)$, reclassifying an equal number to low or high probability of recovery ( $n=52$ and 56 respectively) in stage 2 . These results suggest that the final combined model is slightly better at identifying participants at high and low probability of recovery $(79 \%$ and $75 \%$ of patients in these two groups correctly classified respectively) than the stage 1 model. The combined model misclassifies $18 \%$ of those who did not recover (38/209 would have been incorrectly reassured) and 15\% of those who did recover (35/232 would have been unnecessarily referred).

\section{Discussion}

\section{Summary of findings}

The aim of this study was to investigate whether the use of data from repeated assessments enhanced model fit and prognostic performance of primary care prognostic models, in order to better predict long-term outcomes and reduce uncertainty in clinical decision-making. Such information can support decision making regarding the need for monitoring symptoms, or immediate referral or treatment. The results show that short-term repeat assessment of pain had only slightly better prognostic performance compared to short-term change or baseline only scores in predicting long-term disability improvement. Furthermore, a full baseline prediction model incorporating multiple prognostic factors does not necessarily add prognostic value to a repeat short-term assessment of pain. Using a hypothetical clinical scenario, combining information from a full prediction model assessed at baseline with

Table 3 Strength of association (odds ratios, 95\% Cl), goodness-of-fit statistics, and discrimination for prediction models using a baseline multivariable prognostic model versus baseline pain only, short-term change or repeat score at repeat assessment - long-term perceived recovery as outcome

\begin{tabular}{|c|c|c|c|c|c|c|}
\hline \multirow[t]{2}{*}{ Study author } & \multirow[t]{2}{*}{ Prediction model } & \multirow[t]{2}{*}{ OR $(95 \% \mathrm{Cl})$} & \multicolumn{2}{|c|}{ Goodness-of-fit statistics } & \multirow{2}{*}{$\begin{array}{l}\text { C-statistic } \\
(95 \% \mathrm{Cl})\end{array}$} & \multirow{2}{*}{$\begin{array}{l}\text { Comparison of } \\
c \text {-statistics ( } p \text {-value) }\end{array}$} \\
\hline & & & $\begin{array}{l}\text { Hosmer \& } \\
\text { Lemeshow test }\end{array}$ & $\begin{array}{l}\text { Nagelkerke } \\
\text { pseudo R square }\end{array}$ & & \\
\hline \multirow[t]{2}{*}{ Kuijpers } & $\begin{array}{l}\text { Full model }(n=441) \\
\text { Baseline pain }(0-10) \\
\text { Duration of complaint: } \\
\text { - less than } 5 \text { weeks } \\
\text { - } 6-11 \text { weeks } \\
\text { - more than } 3 \text { m } \\
\text { Concomitant LBP (yes/no) } \\
\text { Shoulder pain score at } \\
\text { physical examination (0-14) } \\
\text { Gradual onset (yes/no) }\end{array}$ & $\begin{array}{l}0.89(0.82 \text { to } 0.98)^{*} \\
1 \\
0.68(0.40 \text { to } 1.18) \\
0.36(0.22 \text { to } 0.60)^{*} \\
0.52(0.32 \text { to } 0.84)^{*} \\
0.96(0.91 \text { to } 1.01) \\
0.60(0.39 \text { to } 0.95)^{*}\end{array}$ & $X_{(8)}^{2}=10.45, p=0.24$ & 0.19 & 0.71 (0.67 to 0.76$)$ & \\
\hline & $\begin{array}{l}\text { Full model plus } 6 \text { w Pain } \\
\text { Score }(n=441)\end{array}$ & $0.72(0.65 \text { to } 0.80)^{*}$ & $X_{(8)}^{2}=10.89, p=0.21$ & 0.29 & 0.78 (0.74 to 0.83 ) & $\begin{array}{l}\text { Full model vs. full } \\
\text { model plus } 6 \mathrm{w} \text { pain } \\
\text { score, } p<0.001^{*}\end{array}$ \\
\hline \multirow[t]{2}{*}{$\begin{array}{l}\text { Van der } \\
\text { Windt }\end{array}$} & $\begin{array}{l}\text { Full model }(n=282) \\
\text { Baseline Pain }(0-10) \\
\text { Co-existing neck pain (yes/no) } \\
\text { Preceding trauma (yes/no) } \\
\text { Diagnosis Acute Bursitis } \\
\text { (yes/no) }\end{array}$ & $\begin{array}{l}0.80(0.69 \text { to } 0.91)^{*} \\
0.47(0.27 \text { to } 0.82)^{*} \\
7.44(1.69 \text { to } 32.85)^{*} 2.32 \\
(1.03 \text { to } 5.20)^{*}\end{array}$ & $X_{(8)}^{2}=9.05, p=0.34$ & 0.17 & 0.72 (0.66 to 0.78 ) & \\
\hline & $\begin{array}{l}\text { Full model plus } 4 \text { w Pain } \\
\text { Score }(n=270)\end{array}$ & $0.85(0.76 \text { to } 0.95)^{*}$ & $X_{(8)}^{2}=15.07, p=0.06$ & 0.22 & 0.75 (0.69 to 0.82$)$ & $\begin{array}{l}\text { Full model vs. full } \\
\text { model plus } 4 \mathrm{w} \\
\text { pain score, } p=0.10\end{array}$ \\
\hline
\end{tabular}


Table 4 Frequencies of low, intermediate and high probability of recovery and observed long-term recovery when classifying shoulder pain patients at baseline based on the full prediction model

\begin{tabular}{|c|c|c|c|c|}
\hline \multirow[t]{2}{*}{ Hypothetical scenario } & \multirow[t]{2}{*}{ Classification } & \multirow{2}{*}{$\begin{array}{l}\text { Frequency } \\
n(\%)\end{array}$} & \multicolumn{2}{|c|}{ Perceived recovery at six months } \\
\hline & & & $\begin{array}{l}\text { Not recovered } \\
N=209 n(\%)\end{array}$ & $\begin{array}{l}\text { Recovered } \\
N=232 n(\%)\end{array}$ \\
\hline \multirow[t]{3}{*}{ Stage 1 Full prediction model at baseline $(n=441)$} & 1 - Low probability of recovery & $87(19.7)$ & $64(73.6)$ & $23(26.4)$ \\
\hline & 2 - Intermediate probability of recovery & $229(51.9)$ & $116(50.7)$ & $113(49.3)$ \\
\hline & 3 - High probability of recovery & $125(28.3)$ & $29(23.2)$ & $96(76.8)$ \\
\hline \multirow{3}{*}{$\begin{array}{l}\text { Stage } 1 \& 2 \text { Full model plus re-assessment of pain } \\
\text { at six weeks - Intermediate group only }(n=229)\end{array}$} & 1 - Low probability of recovery & $52(22.7)$ & $40(76.9)$ & $12(23.1)$ \\
\hline & 2 - Intermediate probability of recovery & $121(52.8)$ & $67(55.4)$ & $54(44.6)$ \\
\hline & 3 - High probability of recovery & $56(24.5)$ & $9(16.1)$ & $47(83.9)$ \\
\hline \multirow[t]{3}{*}{$\begin{array}{l}\text { Model Stage } 1 \& 2 \text { Full model plus re-assessment } \\
\text { of pain at six weeks }(n=441)\end{array}$} & 1 - Low probability of recovery & $\begin{array}{l}87+52= \\
139(31.5)\end{array}$ & $\begin{array}{l}64+40= \\
104(74.8)\end{array}$ & $\begin{array}{l}23+12= \\
35(25.2)\end{array}$ \\
\hline & 2 - Intermediate probability of recovery & $121(27.4)$ & $67(55.4)$ & $54(44.6)$ \\
\hline & 3 - High probability of recovery & $\begin{array}{l}125+56= \\
181(41.0)\end{array}$ & $\begin{array}{l}29+9= \\
38(21.0)\end{array}$ & $\begin{array}{l}96+47= \\
143(79.0)\end{array}$ \\
\hline
\end{tabular}

a brief repeat assessment of pain in only those with uncertain prognosis at initial assessment may provide an efficient and effective strategy towards reducing uncertainty and improving discrimination between those at high or low probability of long-term recovery from a painful shoulder condition.

\section{Comparison with existing literature}

The results of this study do partially confirm those of two similar studies $[8,11]$ which also examined the added prognostic value of short-term monitoring on longer-term outcomes. Dunn \& Croft [11] used the same dataset as one of those used in the present study, and found that repeat assessment of other prognostic factors (e.g. fear-avoidance beliefs) led to an improvement in predictions longer-term. The similarity between this previous study and the findings from the other datasets included here strengthens the argument that repeat assessment can provide additional prognostic information across different outcome measures. Wand et al. [8] found that a 'subacute profile' (predictors measured at six weeks) was the best at predicting long-term disability compared to an 'acute profile' (baseline scores only) and compared to change between baseline and six-week assessment. Our study extended the findings of both of these studies by testing a possible clinical scenario in which a more efficient strategy (only re-assessing those with uncertain prognosis rather than the entire sample) could be applied.

The highest c-statistics obtained in objective 2 are not as high as those reported for other prognostic models [2-5]. For example, the STarT Back tool which can be used to stratify patients with low back pain [24] has demonstrated better prognostic performance (e.g. [25, 26]). Variability in performance can be explained by differences in the outcome measure used in each study (function versus recovery), prognostic factors included in the model, the measures used to assess prognostic factors, or differences in the study population. However, these other studies could not be used as they did not include a short-term assessment point which we required in the present analysis. The aim of our analysis was not to find the "best" model, but rather to find the most appropriate studies to test our hypotheses and clinical scenario for incorporating repeat assessment. Further research is required to confirm whether this improvement holds for other baseline prognostic models, regardless of prognostic performance.

\section{Strengths and Limitations}

This study included four relatively large ( $n$ at least 512) datasets covering two common musculoskeletal complaints. The studies included different measures of disability and used slightly different measurement time points, but the results overall were very similar, which gives strength to the study findings [27]. The number of cases included in each of the analyses did differ, due to dropout over time in each of the studies. Imputation was considered, but a sensitivity analysis using complete case data did not result in any significant differences in results. The sample sizes available for each of the datasets included in this study did meet guidance around numbers of cases required for building prognostic models of an event to predictor ratio of at least 10 (e.g. [28]), although we realise that such guidance may not be entirely suitable for the specific analysis performed in this study.

The inclusion of different musculoskeletal pain regions could be considered problematic as shoulder pain problems may have a different rate of recovery, and different prognostic factors compared to back pain problems. However, recent cohort studies and systematic reviews have highlighted similarities in symptom trajectories and 
identified generic prognostic factors across different regional musculoskeletal pain problems (e.g. $[6,29,30])$. This is supported by our findings from objective 1, confirming the prognostic value of short-term changes in pain for predicting long-term disability outcomes in both back and shoulder pain.

The included studies were selected according to specific criteria to test the present study hypothesis, but were not systematically searched for. This means that we may have missed additional studies that could have been included in our analysis. However, a search of the wider literature found that very few prognosis studies set in primary care include a short-term assessment point, which was a requirement for our analysis. We would however welcome further analysis of the prognostic value of repeat pain assessment in other settings or populations.

Not every dataset could be used for analysis of each objective. While both the Van der Windt and Kuijpers datasets included a prognostic model, differences between these models in terms of the proportion of participants recovered (outcome event rate) would require different classifications for predicted probabilities in order to address objective 3, making them difficult to compare. Only the Kuijpers model was therefore used for this analysis to provide an example for how such a strategy may be used in clinical practice and how researchers can investigate prognosis in the future. To strengthen these findings, replication in other datasets and other musculoskeletal conditions is needed, perhaps especially because prognostic performance of the models presented here could be considered as moderate at best.

The datasets examined here only contain patients who consulted for musculoskeletal pain, and provided data at both baseline and follow-up, and therefore may not be representative of people who have musculoskeletal pain but choose not to consult, or would not re-consult when invited for a repeat assessment. The datasets included in this study did not all take baseline assessments at the point of consultation. As most patients are likely to consult when their pain is at its worst, and may therefore experience a natural reduction in pain shortly after consulting, the scores obtained in these studies may give a different interpretation and prognostic value than if scores were obtained during the consultation. Studies investigating prognostic factors at the point of care found that pain reduced shortly following consultation, [31] suggesting that the point at which measures are taken is important and could affect the accuracy of any prediction models derived [31].

The clinical scenario presented here does not offer optimum prediction. However, this version of the scenario (with three equal risk categories), while realistic for this example, was meant as an illustration of this approach; it could be that different cut-off points for low, intermediate and high probability would result in fewer people being asked to re-consult. The choice of cut-off point should therefore depend on clinically relevant thresholds for treatment and referral. The number of patients in the intermediate group at baseline who needed to return for repeat assessment was still large in our scenario, highlighting the large amount of uncertainty in the prognosis of musculoskeletal pain conditions. Similar proportions for intermediate groups were used in the STarT Back study, [24] which included active management of this group rather than a 'watch and wait' scenario, although in the STarT Back study these proportions were a reflection of the patients included rather than prespecified proportions as in the present study. The scenario presented here builds on this by ensuring that the lowand high-probability groups are optimally identified, either at first consultation or within a few weeks of that first consultation. It could be that a stronger prognostic model with better prognostic performance is needed in order to more clearly differentiate between patients who do or do not require referral.

The hypothetical scenario itself, while aiming to reduce the number of inappropriate clinical decisions, may also lead to an increase in consultations with an impact on GP time and costs. It could be that the single question about pain could be asked via a phone call, SMS text message/smart phone application 4-6 weeks after the initial consultation, with only those who still report pain at that point being invited back to see their GP.

\section{Conclusions}

This study investigated a clinical scenario (Fig. 1) that could be used to help reduce the uncertainty of clinical decisionmaking in GP consultations with a patient with musculoskeletal pain. This scenario shows a decrease in unnecessary referrals by proposing a brief (single question), short-term repeat assessment in those where prognosis remains uncertain at the first consultation. It should also be acknowledged that such a strategy has costs too, such as potential delay in treatment, cost of repeat assessment, and the need to have a system for combining baseline and repeat assessment measures, which might make such a strategy unsuitable in some circumstances. However, the importance of this study lies in its shift towards reducing uncertainty and enabling clinicians to more confidently refer or reassure people at an early stage using minimal information.

Prognostic models would potentially benefit from the inclusion of short-term assessments of key prognostic factors, in addition to baseline assessments. Further research should investigate to what extent the proposed strategy improves management compared to more extensive data collection at a single point in time (baseline), across different health conditions, in order to further test the added value of monitoring for estimating prognosis and optimising clinical decision making. 


\section{Additional file}

Additional file 1: Changes over time (Means, SDs and percentages) and descriptive data for outcome and predictor variables. (DOCX $21 \mathrm{~kb}$ )

\section{Acknowledgements}

We would like to thank Joie Ensor who provided statistical advice on how to carry out the comparisons of the c-statistics, and the reviewers who provided helpful comments to improve the manuscript.

\section{Funding}

The National Institute for Health Research School for Primary Care Research (NIHR SPCR) is a partnership between the universities of Birmingham, Bristol, Keele, Manchester, Nottingham, Oxford, Southampton and University College London. This paper presents independent research funded by the National Institute for Health Research School for Primary Care Research. The views expressed are those of the authors and not necessarily those of the NHS, the NIHR or the Department of Health.

DvdW received funding from an MRC Partnership Grant for the PROGnosis RESearch Strategy (PROGRESS) group (grant reference number: G0902393). GM and DvdW are supported by an Arthritis Research UK Centre for Primary Care (Grant Number 20202).

DL is supported by the NIHR Oxford Biomedical Research Centre

\section{Availability of data and materials}

The datasets analysed during the current study are available from the corresponding author on reasonable request.

\section{Author's contributions}

DvdW conceived the idea for the study. GM, KJ and DvdW analysed the data. GM, KJ, DvdW, GP, KD, TK, DL and IS provided interpretation of the findings. GM, KJ, DvdW, GP, KD, TK, DL and IS read and approved the final manuscript.

\section{Competing interests}

The authors declare that they have no competing interests.

\section{Consent for publication}

Not applicable.

\section{Ethics approval and consent to participate}

The present report presents secondary analyses of previously published data and ethical approval was therefore not required.

\section{Publisher's Note}

Springer Nature remains neutral with regard to jurisdictional claims in published maps and institutional affiliations.

\section{Author details}

${ }^{1}$ Arthritis Research UK Primary Care Centre, Research Institute for Primary Care and Health Sciences, Keele University, Keele, Staffordshire ST5 5BG, UK. ${ }^{2}$ Nuffield Department of Medicine, University of Oxford and NIHR Oxford Biomedical Research Centre, John Radcliffe Hospital, Oxford, UK. ${ }^{3}$ Department for Guideline Development, The Dutch College of General Practitioners, PO Box 32313502 GE Utrecht, The Netherlands. ${ }^{4}$ Centre for Physical Therapy and Rehabilitation, Geldrop, The Netherlands.

Received: 14 September 2016 Accepted: 24 March 2017 Published online: 04 April 2017

\section{References}

1. Jordan KP, Kadam UT, Hayward R, Porcheret M, Young C, Croft P. Annual consultation prevalence of regional musculoskeletal problems in primary care: an observational study. BMC Musculoskelet Disord. 2010;11:144.

2. Bot SDM, van der Waal JM, Terwee CB, van der Windt DA, Scholten RJ, Bouter LM, Dekker J. Predictors of outcome in neck and shoulder symptoms: a cohort study in general practice. Spine. 2005;30(16):459-70.

3. Dunn KM, Jordan K, Croft PR. Characterizing the course of low back pain: a latent class analysis. Am J Epidemiol. 2006;163(8):754-61.
4. Jellema P, van der Horst HE, Vlaeyen JWS, Stalman WAB, Bouter LM, van der Windt DAWM. Predictors of outcome in patients with (sub)acute low back pain in general practice: patients with a favourable outcome differ across treatment groups. Spine. 2006;31:1699-705.

5. van der Waal JA, Bot SDM, Terwee CB, van der Windt DA, Scholten RJ, Bouter LM, Dekker J. The course and prognosis of knee complaints in general practice. Arthritis Rheum. 2005;53:920-30.

6. Mallen CD, Peat G, Thomas E, Dunn KM, Croft PR. Prognostic factors for musculoskeletal pain in primary care: A systematic review. Br J Gen Pract. 2007;57:655-61.

7. LeBoeff-Yde C, Axén I, Jones JJ, Rosenbaum A, Løvgren PW, Halasz L, Larsen K. The Nordic back pain subpopulation program: the long-term outcome pattern in patients with low back pain treated by chiropractors in Sweden. J Manipulative Physiol Ther. 2005;28(7):472-8.

8. Wand BM, McAuley JH, Marston L, De Souza LH. Predicting outcome in acute low back pain using different models of patient profiling. Spine. 2009;34(18):1970-5.

9. Coste J, Delecoeuillerie G, Delara AC, Leparc JM, Paolaggi JB. Clinical course and prognostic factors in acute low-back-pain - An inception cohort study in primary-care practice. BMJ. 1994;308(6928):577-80.

10. da C Menezes Costa L, Maher CG, Hancock MJ, McAuley JH, Herbert RD, Costa LOP. The prognosis of acute and persistent low-back pain: A metaanalysis. CMAJ. 2012;184(11):E613-24.

11. Dunn KM, Croft PR. Repeat assessment improves the prediction of prognosis in patients with low back pain in primary care. Pain. 2006;126(1-3):10-5.

12. Roland M, Morris R. A study of the natural history of back pain. Part I: Development of a reliable and sensitive measure of disability in low-back pain. Spine. 1983;8(2):141-4.

13. van der Heijden GJMG, Leffers $P$, Bouter LM. Shoulder disability questionnaire design and responsiveness of a functional status measure. J Clin Epi. 2000;53:29-38.

14. Hay E, Thomas E, Paterson S, Dziedzic K, Croft P. A pragmatic randomised controlled trial of local corticosteroid injection and physiotherapy for the treatment of new episodes of unilateral shoulder pain in primary care. Ann Rheum Dis. 2003;62(5):394-9.

15. Jordan KJ, Dunn KM, Lewis M, Croft P. A minimal clinically important difference was derived for the Roland-Morris Disability Questionnaire for low back pain. J Clin Epi. 2006:59:45-52.

16. van der Windt DAWM, van der Heijden GJMG, de Winter AF, Koes BW, Devillé W, Bouter LM. The responsiveness of the Shoulder Disability Questionnaire. Ann Rheum Dis. 1998:57:82-7.

17. Hosmer DW, Lemeshow S. Applied logistic regression. New York: Wiley; 2000

18. Cleves MA. From the help desk: Comparing areas under the receiver operating characteristic curves from two or more probit or logit models. Stata J. 2002;2(3):301-13.

19. van der Windt DAWM, Koes B, Boeke AJP, Devillé W, de Jong BA, Bouter LM. Shoulder disorders in general practice: prognostic indicators of outcome. Br J Gen Pract. 1996:46:519-23.

20. Kuijpers T, van der Windt DAWM, Boeke AJP, Twisk JWR, Vergouwe Y, Bouter LM, van der Heijden GJMG. Clinical prediction rules for the prognosis of shoulder pain in general practice. Pain. 2006;120:276-85

21. Field A. Discovering statistics. London: Sage; 2009.

22. Dunn KM, Croft PR. The importance of symptom duration in determining prognosis. Pain. 2006;121:126-32.

23. Swinkels-Meewisse IEJ, Roelofs J, Schouten EGW, Verbeek ALM, Oostendorp $R A B$, Vlaeyen JWS. Fear of movement/(re)injury predicting chronic disabling low back pain: A prospective inception cohort study. Spine. 2006;31(6): 658-64.

24. Hill JC, Whitehurst DGT, Lewis M, Bryan S, Dunn KM, Foster NE, Konstantinou K, Main CJ, Mason E, Somerville S, Sowden G, Vohora K, Hay EM. Comparison of stratified primary care management for low back pain with current best practice (STarT Back): A randomised controlled trial. Lancet. 2011;378: 1560-71.

25. Hill JC, Dunn KM, Lewis M, Mullis R, Main CJ, Foster NE, Hay EM. A primary care back pain screening tool: Identifying patient subgroups for initial treatment. Arthritis Rheum. 2008;59(5):632-41.

26. Mors $\varnothing$ L, Kent P, Albert HB, Hill JC, Kongsted A, Manniche C. The predictive and external validity of the STarT Back Tool in Danish primary care. Eur Spine J. 2013;22:1859-67.

27. Kent PM, Keating JL. Can we predict poor recovery from recent-onset nonspecific low back pain? A systematic review. Man Ther. 2008;13(1):12-28. 
28. Peduzzi P, Concato J, Kemper E, Holford TR, Feinstein AR. A simulation study of the number of events per variable in logistic regression analysis. J Clin Epidemiol. 1996;49(12):1373-9.

29. Henschke N, Ostelo RWJG, Terwee CB, van der Windt DAWM. Identifying generic predictors of outcome in patients presenting to primary care with nonspinal musculoskeletal pain. Arthrit Care Res. 2012;64(8):1217-24.

30. Artus M, Campbell P, Mallen CD, Dunn KM, van der Windt DAW. Generic prognostic factors for musculoskeletal pain in primary care: A systematic review. BMJ Open. 2017;7:e012901.

31. Mallen CD, Thomas E, Belcher J, Rathod T, Croft P, Peat G. Point-of-care prognosis for common musculoskeletal pain in older adults. JAMA Int Med. 2013;173(12):1119-25.

Submit your next manuscript to BioMed Central and we will help you at every step:

- We accept pre-submission inquiries

- Our selector tool helps you to find the most relevant journal

- We provide round the clock customer support

- Convenient online submission

- Thorough peer review

- Inclusion in PubMed and all major indexing services

- Maximum visibility for your research

Submit your manuscript at www.biomedcentral.com/submit
Biomed Central 